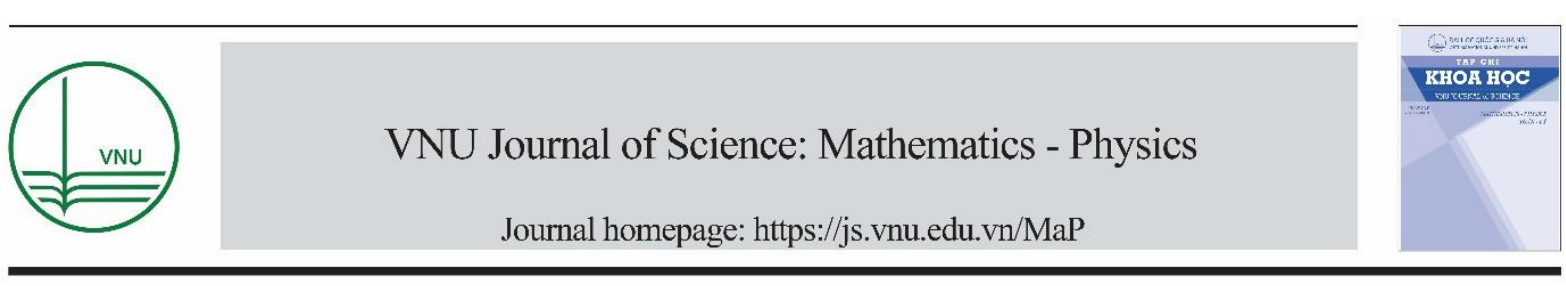

Original Article

\title{
Influence of Confined Phonons on the Hall Coefficient in a Cylindrical Quantum Wire with an Infinite Potential (for Electron-acoustic Optical Phonon Scattering)
}

\author{
Pham Ngoc Thang 1,", Do Tuan Long ${ }^{1}$, Nguyen Quang Bau ${ }^{1}$, Le Thai Hung ${ }^{2}$ \\ ${ }^{1}$ Faculty of Physics, VNU University of Science, 334 Nguyen Trai, Hanoi, Vietnam \\ ${ }^{2}$ VNU University of Education, 144 Xuan Thuy, Hanoi, Vietnam \\ Received 13 March 2019 \\ Revised 15 June 2019; Accepted 01 July 2019
}

\begin{abstract}
This paper studies the influence of confined acoustic phonons on the Hall coefficient $(\mathrm{HC})$ in a cylindrical quantum wire (CQW) with an infinite potential (for electron - confined acoustic phonons scattering). The paper considers the case where CQW is placed in a perpendicular magnetic field $\vec{B}$, a constant electric field $\overrightarrow{E_{1}}$ and an intense electromagnetic wave $\vec{E}=\overrightarrow{E_{0}} \sin \Omega t$. By using the quantum kinetic equation for electrons interacting with confined optical phonons (COP), analytical expressions for $\mathrm{HC}$ are obtained. The application of numerical calculations to $\mathrm{GaAs} / \mathrm{GaAsAl}$ cylindrical quantum wire shows that the $\mathrm{HC}$ depends on magnetic field $\mathrm{B}$, temperature $\mathrm{T}$, frequency $\Omega$ and amplitude $\mathrm{E}_{0}$ of laser radiation and on quantum indices $\mathrm{m}_{1}$ and $\mathrm{m}_{2}$ characterizing the phonon confinement. This influence is due to the quantum indices $\mathrm{m} 1$ and $\mathrm{m}_{2}$ which increase the Hall coefficient by 2.3 times in comparison with the case of unconfined phonons. When the quantum numbers $\mathrm{m}_{1}$ and $\mathrm{m}_{2}$ go to zero, the result is the same as in the case of unconfined phonons.

Keywords: Hall coefficient, quantum kinetic equation, cylindrical quantum wire, confined acoustic phonon.
\end{abstract}

\section{Introduction}

In recent years, the study of low - dimensional semiconductor systems has been increasingly interested, include the electrical, the magnetic and the optical properties. In these systems, the motion of carriers is restricted, thus leading to their new properties under the action of external fields for example: the absorption coefficient of an electromagnetic wave, the Hall effect, the Radioelectric

\footnotetext{
${ }^{*}$ Corresponding author.

Email address: pntm.777@gmail.com
}

https//doi.org/ 10.25073/2588-1124/vnumap.4333 
effect, the Acoustoelectric effect. The Hall effect - the effect of drag of charge carriers caused by the external magnetic field has been studied extensively [1-3]. There have been study of the Hall effect in bulk semiconductor in the presence of electromagnetic waves, in which classical theory of Hall effect in bulk semiconductor when placed in electricity, the magnetic field is perpendicular to the presence of an electromagnetic wave is built on the basis of Boltzman's classical kinetic equation, while quantum theory is based on quantum-kinetic equation [4]. In two-dimensional semiconductor systems, there have been studies on the Hall effect with the electronic - confined phonon scattering [5-9]. In onedimensional semiconductor system, there have been studies on the Hall effect with the confined electronics - unconfined phonon [10]. But the influence of the confined phonons on the $\mathrm{HC}$ in onedimensional semiconductor system is not studied. In this work, we study new properties of the HC under the effect of COP. Considering an infinite potential quantum wire subjected to a dc electric field $\vec{E}$, a magnetic field $\vec{B}$ and a laser radiation $\vec{E}=\overrightarrow{E_{0}} \sin \Omega t$. The article is organized as follows: in section 2 we present the confinement of electron and optical phonons in a CQW. Thus, by using the quantum kinetic equation method, we obtained analytical expressions for the Hall coefficient. Numerical results and discussions for the GaAs/GaAsAlcylindrycal quantum wire are given in section 3. Finally, section 4 shows remarks and conclusions.

\section{The influence of confined phonons on the hall coefficient in a cylindrycal quantum wire with an infinite potential}

Consider a cylindrycal quantum wire with an infinite potential $V=\pi R^{2} L$ subjected is placed in a perpendicular magnetic field $\vec{B}$, a constant - electric field $\overrightarrow{E_{1}}$ and an intense electromagnetic wave $\vec{E}=$ $\overrightarrow{E_{0}} \sin \Omega t$. Under the influence of the material confinement potential, the motion of carriers is restricted in $\mathrm{x}, \mathrm{y}$ direction and free in the $\mathrm{z}$ one. So, the wave function of an electron and its discrete energy now becomes:

$$
\begin{gathered}
\Psi_{\mathrm{n}, \mathrm{l}, \overrightarrow{\mathrm{k}}}(\vec{r}, \Phi, z)=\frac{1}{\sqrt{V_{0}}} e^{i m \Phi} e^{i \vec{k} z} \varphi_{n, l}(r), \text { where } \varphi_{n, l}(r)=\frac{1}{J_{n+1}\left(B_{n, l}\right)} J_{n}\left(B_{n, l} \frac{r}{R}\right) \\
\varepsilon_{n, l}\left(\vec{k}_{z}\right)=\left(N+\frac{n}{2}+\frac{l}{2}+\frac{1}{2}\right) \hbar \omega_{c}+\frac{\hbar^{2} k^{2}}{2 m}-\frac{1}{2 m}\left(\frac{e E 1}{\omega_{c}}\right)^{2}
\end{gathered}
$$

where $k, m$ is the wave vector and the effective mass of an electron, $\mathrm{R}$ being the radius of the CQW, $n=1,2,3, \ldots$ and $l=0, \pm 1, \pm 2, \ldots$ being the quantum numbers charactering the electron confinement, $\hbar$ is the Planck constant, $\omega_{c}=\frac{e B}{m}$ is the cyclotron frequency.

When phonons are confined in CQW, the wave vector and frequency of them are given by [11,12]:

$$
\vec{q}=\left(\vec{q}_{m_{1} m_{2}}, q_{z}\right), \omega_{m_{1}, m_{2}, \vec{q}_{\perp}}=\sqrt{\omega_{0}^{2}-\beta^{2}\left(q_{m_{1} m_{2}}^{2}+q_{z}^{2}\right)}
$$

Where $\beta$ is the velocity parameter, $\mathrm{m}_{1}, \mathrm{~m}_{2}=1,2,3, \ldots$ being the quantum numbers charactering phonon confinement. Also, matrix element for confined electron - confined optical phonon interaction in the CQW now becomes [11]

$$
\begin{gathered}
D_{n_{1}, l_{1}, n_{2}, l_{2}, q_{z}}^{m_{1}, m_{2}}=C_{\vec{q}}^{m_{1}, m_{2}} * I_{n_{1}, l_{1}, n_{2}, l_{2}}^{m_{1}, m_{2}} \text { where }\left|C_{\vec{q}}^{m_{1}, m_{2}}\right|^{2}=\frac{\mathrm{e}^{2} \omega_{0}}{2 \varepsilon_{0} V}\left(\frac{1}{\chi_{\infty}}-\frac{1}{\chi_{0}}\right) \frac{1}{\mathrm{q}_{\mathrm{z}}^{2}+\mathrm{q}_{m_{1}, m_{2}}^{2}} \\
\mathrm{I}_{n_{1}, l_{1}, n_{2}, l_{2}}^{m_{1}, m_{2}}=\frac{2}{\mathrm{R}^{2}} \int_{0}^{\mathrm{R}} J_{\left|n_{1}-n_{2}\right|}(q, R) \varphi_{\mathrm{n}_{2}, \mathrm{l}_{2}}^{*}(\mathrm{r}) \varphi_{\mathrm{n}_{1}, \mathrm{l}_{1}}(\mathrm{r}) \mathrm{rdr} .
\end{gathered}
$$

Though equations (1-5), it has been seen that the CQW with new material confinement potential gives the different electron wave function and energy spectrum. In addition, the contribution of 
confined phonon could enhance the probability of electron scattering. As a result, the Hall Coefficient in a CQW under influence of confined optical phonon and laser radiation should be studied carefully to find out the new properties. The effect of confined optical phonons and the laser radiation modify the Hamitonian of the confined electron - confined optical phonons system in the CQW. This leads the quantum kinetic equation for electron distribution. Using Hamiltonian of the confined electrons confined optical phonons in a CQW, we establish the quantum kinetic equation for electron distribution function. After some manipulation, the expression for the conductivity tensor is obtained:

$$
\sigma_{\text {ie }}=\frac{\tau}{1+\omega_{\mathrm{c}}^{2} \tau^{2}}\left\{\delta_{\mathrm{ik}}-\omega_{\mathrm{c}} \tau \varepsilon_{\mathrm{ijk}} \mathrm{h}_{\mathrm{k}}+\omega_{\mathrm{c}}^{2} \tau^{2} \mathrm{~h}_{\mathrm{i}} \mathrm{h}_{\mathrm{j}}\right\}\left\{\mathrm{a} \delta_{\mathrm{ei}}+\mathrm{b}\left(\delta_{\mathrm{je}}-\omega_{\mathrm{c}} \tau \varepsilon_{\mathrm{jef}} \mathrm{h}_{\mathrm{f}}+\omega_{\mathrm{c}}^{2} \tau^{2} \mathrm{~h}_{\mathrm{e}} \mathrm{h}_{\mathrm{f}}\right)\right\}(6)
$$

here $\delta_{i k}$ is the Kronecker delta; $\varepsilon_{i j k}$ being the antisymmetric Levi-Civita tensor; symbols $i, j, k, l, p$ corresponding the components $\mathrm{x}, \mathrm{y}, \mathrm{z}$ of the Cartesian coordinates. From this we obtain the expression for the hall coefficient

$$
\begin{aligned}
& \mathrm{R}_{\mathrm{H}}=-\frac{1}{\mathrm{~B}} \frac{\sigma_{\mathrm{yx}}}{\sigma_{\mathrm{xx}}{ }^{2}+\sigma_{\mathrm{yx}}{ }^{2}} \\
& \text { With } \quad \sigma_{\mathrm{xx}}=\frac{\tau}{1+\omega_{\mathrm{c}}^{2} \tau^{2}}\left\{\mathrm{a}+\mathrm{b}\left[1-\omega_{\mathrm{c}}^{2} \tau^{2}\right]\right\} ; \sigma_{\mathrm{yx}}=\frac{-\tau}{1+\omega_{\mathrm{c}}^{2} \tau^{2}}(\mathrm{a}+\mathrm{b}) \omega_{\mathrm{c}} \tau \\
& a=\frac{L_{z}}{2 \pi} \frac{e \beta \hbar}{m^{2}} \frac{\tau_{0}}{1+\omega_{c}^{2} \tau_{0}^{2}} \exp \left\{\beta\left[\varepsilon_{F}-\hbar \omega_{c}\left(N+\frac{n}{2}+\frac{l}{2}+\frac{1}{2}\right)+\frac{e^{2} E_{1}^{2}}{2 m \omega_{c}^{2}}\right]\right\}\left(\frac{2 m}{\beta \hbar^{2}}\right)^{3 / 2} \frac{\sqrt{\pi}}{2} \\
& \mathrm{~b}=\frac{\mathrm{e}}{\mathrm{m}} \frac{\tau}{1+\omega_{\mathrm{c}}^{2} \tau^{2}} \mathrm{~b}_{0} ; \quad b_{0}=\frac{2 \pi i e}{m} \sum_{\gamma_{1}, \gamma_{2}, m_{1}, m_{2}}\left(I_{\gamma_{1}, \gamma_{2}, m_{2}}^{m_{1},}\right)^{2}\left(A_{1}-A_{2}+A_{3}+A_{4}-A_{5}+A_{6}+A_{7}-A_{8}\right) \\
& A_{1}=\frac{L_{z}}{2 \pi} \frac{m e^{2} \omega_{0}}{2 \varepsilon_{0} V}\left(\frac{1}{X_{\infty}}-\frac{1}{X_{0}}\right) \mathrm{e}^{\beta\left[\varepsilon_{F}-\omega_{c}\left(N_{1}+\frac{n}{2}+\frac{l}{2}+\frac{1}{2}\right)+\frac{1}{2}\left(\frac{e E_{1}}{\omega_{c}}\right)^{2}\right]} \frac{1}{2 \pi} \frac{k_{B} T}{\omega} \\
& {\left[2 q_{m_{1}, m_{2}}^{2} m^{2} A_{11}^{2} \exp \left(-\frac{\beta A_{11}}{2}\right) K_{-2}\left(\frac{\beta A_{11}}{2}\right)+8 A_{11}^{4} m^{4} q_{m_{1}, m_{2}}^{2} \exp \left(-\frac{\beta A_{11}}{2}\right) K_{-3}\left(\frac{\beta A_{11}}{2}\right)\right.} \\
& \left.-m A_{11} \exp \left(-\frac{\beta A_{11}}{2}\right) K_{-1}\left(\frac{\beta A_{11}}{2}\right)-4 m^{3} A_{11}^{3} \exp \left(-\frac{\beta A_{11}}{2}\right) K_{-2}\left(\frac{\beta A_{11}}{2}\right)\right]
\end{aligned}
$$

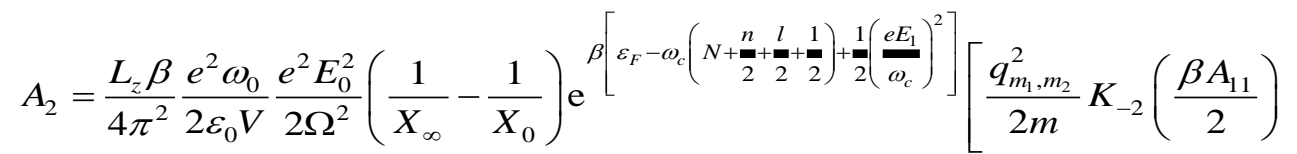

$$
\begin{aligned}
& \left.+\frac{q_{m_{1}, m_{2}}^{2}}{2 m} K_{0}\left(\frac{\beta A_{11}}{2}\right)-A_{11}\left(\frac{\beta A_{11}}{2}\right)-A_{11}\left(\frac{\beta A_{11}}{2}\right)\right] \\
& A_{3}=\frac{L_{z}}{4 \pi} \frac{e^{4} E_{0}^{2} k_{B} T}{8 \varepsilon_{0} V}\left(\frac{1}{X_{\infty}}-\frac{1}{X_{0}}\right) \exp \left\{\beta\left[\varepsilon_{F}-\omega_{\gamma_{1}}-\frac{\hbar^{2} A_{11}}{4}+\frac{\hbar^{2} \Omega}{2}\right]\right\}\left[\Omega K_{0}\left(\frac{\beta \hbar^{2}\left|A_{11}-\Omega\right|}{2}\right)\right. \\
& +\left(\frac{1}{\left|2 m A_{11}-2 m \Omega\right|}\right)^{3 / 2} K_{-3 / 2}\left(\frac{\beta \hbar^{2}\left|A_{11}-\Omega\right|}{2}\right) \Omega q_{m_{1}, m_{2}}^{2}+\frac{q_{m_{1}, m_{2}}^{2}}{2 m} K_{0}\left(\frac{\beta \hbar^{2}\left|A_{11}-\Omega\right|}{2}\right) \\
& -\frac{1}{2}\left|2 m A_{11}-2 m \Omega\right| K_{1}\left(\frac{\beta \hbar^{2}\left|A_{11}-\Omega\right|}{2}\right)-A_{11} K_{0}\left(\frac{\beta \hbar^{2}\left|A_{11}-\Omega\right|}{2}\right)-\left(\frac{1}{\left|2 m A_{11}-2 m \Omega\right|}\right)^{3 / 2} \\
& \left.\left(\Omega q_{m_{1}, m_{2}}^{2}+A_{11} q_{m_{1}, m_{2}}^{2}\right) K_{-3 / 2}\left(\frac{\beta \hbar^{2}\left|A_{11}-\Omega\right|}{2}\right)+\frac{1}{2}\left|2 m A_{11}-2 m \Omega\right| K_{1}\left(\frac{\beta \hbar^{2}\left|A_{11}-\Omega\right|}{2}\right)\right]
\end{aligned}
$$




$$
\begin{gathered}
A_{4}=\frac{m^{2} e^{2} k_{B} T}{2 \varepsilon_{0} V} \beta \frac{e^{2} E_{0}^{2}}{4 m^{2} \Omega^{3}} \frac{L_{z}}{4 \pi^{2}}\left(\frac{1}{X_{\infty}}-\frac{1}{X_{0}}\right) \exp \left[\beta\left(\varepsilon_{F}-\omega_{\gamma_{1}}-\frac{\hbar^{2} A_{11}}{4}-\frac{\hbar^{2} \Omega}{2}\right)\right]\left[\left(q_{m_{1}, m_{2}}^{2}-A_{11}-\Omega\right) K_{0}\right. \\
\left.\left(\frac{\beta \hbar^{2}\left|A_{11}-\Omega\right|}{2}\right)-2 m\left|A_{11}-\Omega\right| K_{1}\left(\frac{\beta \hbar^{2}\left|A_{11}-\Omega\right|}{2}\right)+\left(\Omega q_{m_{1}, m_{2}}^{2}+A_{11} q_{m_{1}, m_{2}}^{2}\right) K_{-1}\left(\frac{\beta \hbar^{2}\left|A_{11}-\Omega\right|}{2}\right)\right] \\
A_{5}=\frac{m^{2} e^{2} k_{B} T}{2 \varepsilon_{0} V} \beta \frac{e^{2} E_{0}^{2}}{4 m^{2} \Omega^{3}} \frac{L_{z}}{4 \pi^{2}}\left(\frac{1}{X_{\infty}}-\frac{1}{X_{0}}\right) \exp \left[\beta\left(\varepsilon_{F}-\omega_{\gamma_{1}}-\frac{\hbar^{2} B_{1}}{2}\right)\right]\left[\frac{\hbar^{2}}{2 m} K_{0}\left(\frac{\beta \hbar^{2}}{2}\right)\right. \\
\left.+\left(\frac{1}{2}-\frac{\hbar^{2} q_{m_{1}, m_{2}}^{2}}{4 m B_{1}}\right) K_{1}\left(\frac{\beta \hbar^{2}}{2}\right)-\frac{q_{m_{1}, m_{2}}^{2}}{4 B_{1}} K_{-2}\left(\frac{\beta \hbar^{2}}{2}\right)\right] \quad \mathrm{K} \text { is Bessel fouction type } 2 \\
A_{6}=\frac{m^{2} e^{2} k_{B} T}{2 \varepsilon_{0} V} \beta \frac{e^{2} E_{0}^{2}}{4 m^{2} \Omega^{4}} \frac{L_{z}}{4 \pi^{2}}\left(\frac{1}{X_{\infty}}-\frac{1}{X_{0}}\right) \exp \left[\beta\left(\varepsilon_{F}-\omega_{\gamma_{1}}-\frac{\hbar^{2} B_{1}}{2}\right)\right] \\
\left.\frac{\hbar^{2} B_{1}}{2 m} K_{1}\left(\frac{\beta B_{1}}{2}\right) \frac{\hbar^{2} q_{m_{1}, m_{2}}^{2}}{2 m} K_{0}\left(\frac{\beta B_{1}}{2}\right)-B_{1} q_{m_{1}, m_{2}}^{2}\left(\frac{1}{2 B_{1}}\right)^{3 / 2} K_{-3 / 2}\left(\frac{\beta B_{1}}{2}\right)\right] \\
A_{7}=\frac{e^{2} k_{B} T}{2 \varepsilon_{0} V} \beta \frac{e^{2} E_{0}^{2}}{4 \Omega^{4}} \frac{L_{z}}{4 \pi^{2}}\left(\frac{1}{X_{\infty}}-\frac{1}{X_{0}}\right) \exp \left\{\beta\left[\varepsilon_{F}-\omega_{\gamma_{1}}+\frac{\beta}{2}\left(B_{1}+\Omega\right)\right]\right\}\left[\frac{\hbar^{2}}{2 m} m\left|B_{1}-\Omega\right| K_{1}\right. \\
\left(\beta\left|B_{1}-\Omega\right|\right)+\left(\Omega-B_{1}-\frac{\hbar^{2} q_{m_{1}, m_{2}}^{2}}{2 m}\right) K_{0}\left(\beta\left|B_{1}-\Omega\right|\right)+q_{m_{1}, m_{2}}^{2}\left(\Omega-B_{1}\right) * \frac{1}{m\left|B_{1}-\Omega\right|} K_{-1}\left(\beta\left|B_{1}-\Omega\right|\right) \\
A_{11}=\varepsilon_{\gamma_{2}}-\varepsilon_{\gamma_{1}}+\omega_{m_{1}, m_{2}, q_{z}} ; \\
A_{8}=\frac{e^{4} k_{B} T}{2 \varepsilon_{0} V} \beta \frac{E_{0}^{2}}{4 \Omega^{3}} \frac{L_{z}}{4 \pi^{2}}\left(\frac{1}{X_{\infty}}-\frac{1}{X_{0}}\right) \exp \left\{\beta\left[\varepsilon_{F}-\omega_{\gamma_{1}}+\frac{\beta}{2}\left(B_{1}-\Omega\right)\right]\right\}\left\{-\hbar^{2}\left(B_{1}+\Omega\right)\right. \\
\left.\beta\left(B_{1}+\Omega\right)\right]-\left(\Omega+B_{\gamma_{2}}-\varepsilon_{\gamma_{1}}-\omega_{m_{1}, m_{2}, q_{z}} ;\right. \\
\Omega
\end{gathered}
$$

The expression (7) is analytics expression of the Hall coefficient in CQW with an infinite potential (for electron - confined optical phonons scattering). From this expression we see, the HC dependent on the magnetic field $\mathrm{B}$,frequency $\Omega$ and amplitude $\mathrm{E}_{0}$ of laser radiation, temperature $\mathrm{T}$ of system and specially the quantum numbers $\mathrm{m}_{1}, \mathrm{~m}_{2}$ characterizing the phonon confinement effect. Where $\mathrm{m}_{1}, \mathrm{~m}_{2}$ goes to zero, we obtain results as case of unconfined phonons [10].

\section{Numerical results and discussions}

In this section, we present the numerical evaluation of the Hall conductivity and the $\mathrm{HC}$ for the $\mathrm{GaAs} / \mathrm{GaAsAl}$ quantum wire. Parameters used in this according to the result in Ref. [11,12]: $m_{e}=$ $0.067 m_{0},\left(m_{0}\right.$ is the free mass of an electron $), \chi_{\infty}=10.9, \chi_{0}=12.9, \varepsilon_{F}=8.10^{-21} \mathrm{~J}, \tau=10^{-12} \mathrm{~s}$, 
$v=8.73 \times 10^{4} \mathrm{~ms}^{-1}, \hbar \omega_{0}=36.25 \mathrm{meV}, \mathrm{V}=1, E_{0}=10^{5} \mathrm{~V} / \mathrm{m}, E_{1}=5.10^{5} \mathrm{~V} / \mathrm{mc}=3.10^{8} \frac{\mathrm{m}}{\mathrm{s}},$, $k_{B}=1.38 \cdot 10^{-23} \mathrm{~J} / \mathrm{K}$

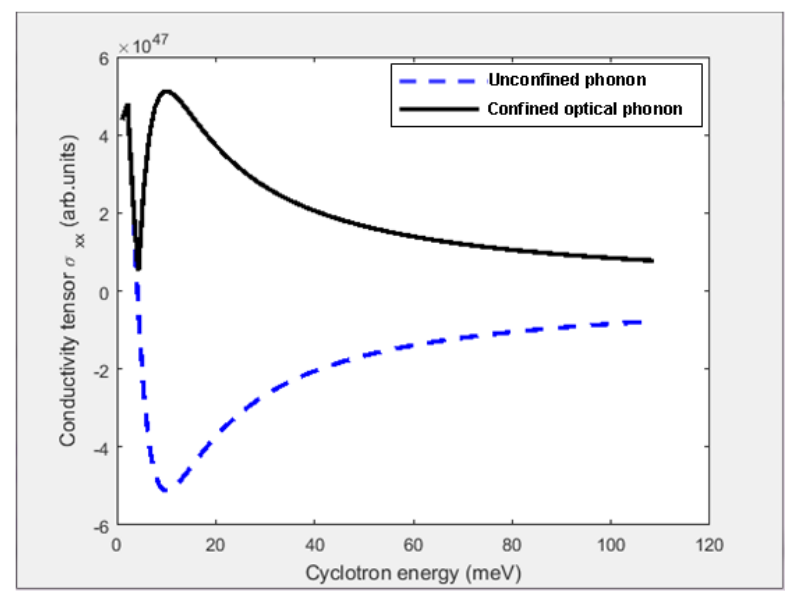

Figure 1.The dependence of the conductivity tensor $\sigma_{x x}$ on the cyclotron energy for confined phonon (solid curve) and unconfined phonon (dashed curve), here $E_{1}=5.10^{5} \mathrm{~V} / \mathrm{m}$ and $L=30 \mathrm{~nm}$

In figure 1, we can see clearly the appearance of oscillations and oscillations are controlled by the ratio of the Fermi energy and energy of cyclotron. First, phonons are confined in 2 dimensions $x, y$, only motion free in the $\mathrm{z}$ one (quantum wires), therefore, The power spectrum of the external phonon depends on the normal effects of free movement, depending on the confined index of phonon $\mathrm{m}_{1}, \mathrm{~m}_{2}$ corresponding to the $\mathrm{x}$ and $\mathrm{y}$ directions. In case confined phonon get more two resonance peaks comparing with that in case of unconfined phonons. When phonons are confined, specially the confined optical phonons frequency is now modified to $\omega_{m_{1}, m_{2}, \vec{q}}=\sqrt{\omega_{0}^{2}-\beta^{2}\left(q_{z}^{2}+q_{m_{1}, m_{2}}^{2}\right)}$. Hence, confined optical phonons make remarkable contribution on the resonance condition.

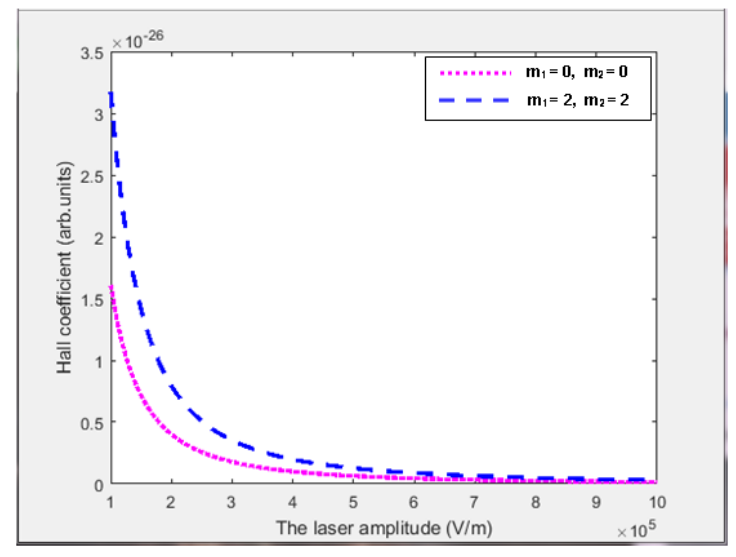

Figure 2. The dependence of the Hall coefficient on the laser amplitude for unconfined phonon $\quad m_{l}=0, m_{2}=0$ (dotted curve) and confined phonon $m_{1}=2, m_{2}=2$ (dashed curve)

Figure 2 shows the nonlinear dependence of the Hall coefficient on the laser amplitude at different values of number $m_{1}, m_{2}$ characterizing the phonon confinement. When the laser amplitude has been 
valid small, which makes an increase of Hall coefficient by 2,3 times in comparison with the case of unconfined phonons. It has been seen that the HC decreases as the increasing of the laser amplitude and the $\mathrm{HC}$ reaches saturation when this amplitude is large. When the quantum number $\mathrm{m}_{1}$ and $\mathrm{m}_{2}$ goes to zero, the result is the same as in the case of unconfined phonons [10].

\section{Conclusions}

In this article, the influence of confined optical phonons on the Hall coefficient in a quantum wires with infinite potential (for electron - confined optical phonons scattering) has been theoretically studied base on quantum kinetic equation method. We obtained the analytical expression of the Hall coefficient in the CQW under the influence of COP. Numerical calculations are also applied for $\mathrm{GaAs} / \mathrm{GaAsAlcylindrycal}$ quantum wire, we see theHCdepends on magnetic field $\mathrm{B}$, temperature $\mathrm{T}$, frequency $\Omega$ and amplitude $E_{0}$ of laser radiation and especially quantum index $m_{1}$ and $m_{2}$ characterizing the phonon confinement. This influence is due to the quantum index $m_{1}$ and $m_{2}$, which makes an increase of Hall coefficient by 2,3 times in comparison with the case of unconfined phonons.

\section{Acknowledgments}

This work was completed with financial support from the QG.17.38

\section{References}

[1] N.Q. Bau, B.D. Hoi, Dependence of the Hall Coefficient on Doping Concentration in Doped Semiconduction, Integrated Ferroelectrics 155 (2014) 39 - 44.

[2] E.H. Hwang, S.D. Sarma, Hall coefficient and magnetoresistance of two-dimensional spin-polarized electron systems, Phys. Rev. B. 73, 121309 (2006) 1 - 4.

[3] G.M. Shmelev, G.I. Tsurkan, N.H. Shon, The magnetoresistance and the cyclotron resonance in semiconductors in the presence of strong electromagnetic wave, Sov. Phys. Semicond., 15 (1981) 156 - 161.

[4] E.M. Epshtein, G.M. Smelev, G.I. Tsurkan, Photostimulated Progresses in Semiconductors. Izd. Shtiinza, Kishinev, Russian, 1987.

[5] N.Q. Bau, D.T. Long, Influence of confined optical phonons and laser radiation on the hall effect in a compositional supperlattice, Physica B 512 (2018) 149 - 154.

[6] P.N. Thang, L.T. Hung, N.Q. Bau, Influence of Confined Acoustic Phonons on the Shubnikov-deHaas Magneto resistance Oscillations in a Doped Semiconductor Superlattice, World Academy of Science, Engineering and Technology International Journal of Physical and Mathematical Sciences 11 (8) (2017) 367-370

[7] J.S. Bhat, B.G. Mulimani, S.S. Kubakaddi, Electron-confined LO phonon scattering rates in GaAs/AlAs quantum wells in the presence of a quantizing magnetic field, Semicond. Sci. Technol. 8 (1993) 1571 - 1574.

[8] S. Rudin, T. Reinecke, Electron-LO-phonon scattering rates in semiconductor quantum wells, Phys. Rev. B. 41 (1990) 7713 - 7717.

[9] D.T. Long, L.T. Hung, N.Q. Bau, Progress in Electromagnetic Research Symposium (PIERS) (2016) 3878.

[10] N.T. Huong, N.Q. Bau, N.V. Nhan,The photostimulated hall effect in a cylindrycal quantum wire with confined Electrons-optical phonons scattering, Journal of Military Science and Technology Research, 3 (45) (2016) 131 - 139.

[11] A. Zou, H. Xie, Effects of confined LO and SO phonon modes on polaron in freestanding cylindrical quantum wire with parabolic confinement, Modern Physics Letters B. 23 (2009) 3515 - 3523.

[12] X.F. Wang, X.L.Lei, Polar - optic phonons and high - field electron transport in cylindrical GaAs/AlAs quantum wires, Phys. Rev. B. 49 (1994) 4780 - 4789. 